\title{
The evolving role of lymphadenectomy for bladder cancer: why, when, and how
}

\author{
Vignesh T. Packiam, Matvey Tsivian, Stephen A. Boorjian \\ Department of Urology, Mayo Clinic, Rochester, MN, USA \\ Contributions: (I) Conception and design: All authors; (II) Administrative support: SA Boorjian; (III) Provision of study materials or patients: All \\ authors; (IV) Collection and assembly of data: All authors; (V) Data analysis and interpretation: All authors; (VI) Manuscript writing: All authors; (VII) \\ Final approval of manuscript: All authors. \\ Correspondence to: Stephen A. Boorjian. Department of Urology, Mayo Clinic, 200 1st St. Southwest, Rochester, MN 55905, USA. \\ Email: Boorjian.stephen@mayo.edu.
}

\begin{abstract}
Radical cystectomy $(\mathrm{RC})$ represents a standard treatment for non-metastatic muscle-invasive and select high-risk non-muscle invasive bladder cancer. Lymphadenectomy performed at time of RC identifies nodal metastases in up to $25 \%$ of patients despite normal imaging. There has been an increasing utilization of pelvic lymph node dissection (PLND) with RC since 1950, and in fact lymph node dissection is now recommended in contemporary National Comprehensive Cancer Network (NCCN) guidelines. Benefits of removing of nodal disease include improved staging, guidance for adjuvant treatment, and potentially improved oncologic outcomes. Advantages of dissection have been suggested among both node-negative and node-positive patients. Numerous studies have attempted to define the optimal dissection characteristics of lymphadenectomy with regard to nodal yield and anatomic boundaries of dissection. The ideal extent of lymphadenectomy remains uncertain due to the retrospective and non-randomized nature of the majority of existing reports, which are thereby limited by significant confounding and selection bias. Two randomized controlled trials have investigated this issue, one of which LEA AUO AB 25/02 recently reported its outcomes, demonstrating no significant improvement in 5-year outcomes with an extended dissection. Meanwhile, the Southwest Oncology Group 1011 trial has completed enrollment and data are maturing. While current data preclude definitive recommendations, herein we review the why, when, and how to perform a PLND for bladder cancer.
\end{abstract}

Keywords: Bladder cancer; radical cystectomy (RC); lymph node dissection; lymphadenectomy

Submitted Dec 26, 2018. Accepted for publication May 30, 2019.

doi: $10.21037 /$ tau.2019.06.01

View this article at: http://dx.doi.org/10.21037/tau.2019.06.01

\section{Introduction}

There were an estimated 549,393 newly diagnosed cases of bladder cancer and 199,922 bladder cancer deaths worldwide in 2018 (1). Approximately 30\% of patients with bladder cancer present with muscle-invasive disease (2). Radical cystectomy (RC) with pelvic lymph node dissection (PLND) is a standard treatment for patients with muscleinvasive bladder cancer as well as for patients with nonmuscle invasive bladder cancer that is endoscopically unresectable or unresponsive to intravesical therapy (3-6).
Despite normal preoperative imaging, $8 \%$ and $25 \%$ of patients undergoing RC for non-muscle invasive and muscle-invasive bladder cancer, respectively, have been reported to have pathologically involved lymph nodes at the time of PLND $(7,8)$. Indeed, current National Comprehensive Cancer Network (NCCN) guidelines advocate that a lymph node dissection be performed with RC (3). This recommendation is based on existing observational data suggesting improved risk stratification and oncologic outcomes for patients who undergo PLND versus those who do not (9). At the same time, data remain 
mixed and therefore consensus undefined on the optimal extent of PLND (10).

\section{History of lymphadenectomy for bladder cancer}

The concept of lymph node dissection for malignancy is rooted in 1886, when Halstead reported a survival benefit for patients with breast cancer who underwent regional lymphadenectomy at time of mastectomy (11). En bloc regional lymphadenectomy for bladder cancer was reported by Leadbetter in 1950, who postulated that the relatively high death rate from "palliative" cystectomy alone was due to unrecognized malignancy in pelvic lymphatic tissue (12). Several subsequent early series noted that the additional dissection with PLND minimally changed the morbidity profile of cystectomy $(13,14)$. Interestingly, over the next decades, survival dramatically improved in node-positive patients, from $<1 \%$ to $36 \%(13,15)$. The utilization of PLND has in fact continued to rise; for example, an analysis of the Surveillance, Epidemiology, and End Results (SEER) database by Cole $e t$ al. revealed that PLND increased from $26 \%$ in 1998 to $61 \%$ in 2010 (16).

\section{Rationale for PLND}

PLND with RC may confer several benefits. First, PLND provides the most accurate staging and prognostic information which in turn facilitates risk stratification and patient counseling. Second, PLND identifies patients with involved nodes who may be candidates for adjuvant therapies (17-20). Moreover, removing involved nodes may itself improve oncologic control.

With regard to staging, the American Joint Committee on Cancer (AJCC) TNM staging system classifies bladder cancer nodal stage as follows: N1-a single lymph node metastasis in the true pelvis (peri-vesical, obturator, internal and external iliac, or sacral lymph nodes); N2multiple lymph node metastases in the true pelvis; N3common iliac lymph node metastases $(21,22)$. Of note, M1a designates lymph node metastases proximal to the common iliac vessels, which would correspond to nodes dissected by Level 3 or super-extended templates (see below), although some major centers argue these should still be considered regional nodes $(23,24)$. The prognostic utility of this system has been confirmed in various studies (25).

Determining lymph node involvement also enhances our ability to establish prognosis. An increasing number of overall positive nodes has been associated with poorer survival, with a wide range of prognostic thresholds suggested $(18,26)$. Lymph node density, or ratio of positive lymph nodes, is the proportion of positive nodes to overall nodes removed. This measure somewhat accounts for extent of dissection and rigor of pathologic examination (27). Some series reported decreased survival with a lymph node density $>20 \%$, with 5 -year overall survival (OS) dropping from $64 \%$ to $8 \%(27,28)$. Finally, the presence of extracapsular nodal extension (ECE) has been demonstrated to be associated with decreased cancer specific survival in institutional series and meta-analysis (29-31). Accurate identification of node-positive disease may also guide the administration of adjuvant therapy, which in turn may further improve survival. Indeed, guidelines recommend that patients who did not receive neoadjuvant chemotherapy and are found to have node-positive disease at cystectomy undergo adjuvant chemotherapy or enroll in clinical trials (3).

Perhaps the topic of greatest controversy with regard to the potential benefits of PLND is the ability of PLND to independently improve oncologic control. Notably, a survival benefit has been demonstrated with an increasing number of nodes removed as well as larger dissection templates in various series to date $(9,17-20,32)$. The benefits from PLND have been observed in both pathologically node-negative and node-positive patients (18). In nodenegative patients, the survival benefits conferred by PLND may be due to both elimination of micro-metastatic disease and by accurate prognostic staging, also known as the Will Rogers phenomenon (33). That is, node-negative group with a PLND would have decreased "contamination" with node-positive patients, and the node-positive group would include patients with a lower burden of metastatic disease. In node-positive patients, removal of involved nodes may also serve to debulk disease and improve the efficacy of adjuvant therapy, and be curative in select patients for whom all metastatic disease is resected. Since the majority of these observations are made in non-randomized series, confounding by patient factors and quality of operation that is associated with increasing node yield must be acknowledged.

\section{When to perform a PLND?}

The NCCN guidelines state to perform a bilateral PLND at time of RC, and to include at least the common iliac, internal iliac, external iliac, and obturator nodes (3). Contemporary clinical staging and prognostic criteria do not currently allow for selective or risk-adapted PLND. 
Indeed, recent assessment of 1,016 patients attempted to predict nodal metastases based on preoperative pathologic and radiologic factors but showed that none were predictive of nodal involvement (34). Current radiographic staging predominantly includes computed tomography (CT) and magnetic resonance imaging (MRI), with positron emission tomography (PET)/CT scans utilized selectively as well. However, these modalities all have been found to have poor sensitivity for detecting lymph node metastases (35-39). Moreover, positive lymph nodes are identified in approximately $8 \%$ of those with non-muscle invasive bladder cancer and $25-30 \%$ of those with muscle-invasive bladder cancer without evidence of nodal metastases on $\mathrm{CT}$ scan, suggesting that even patients undergoing $\mathrm{RC}$ for non-muscle invasive disease, as well as patients with what is assessed as a "negative" staging evaluation, merit PLND $(7,8,30)$. A study assessing 276 patients with preoperative CT reported accuracy in predicting lymph node metastases of $54 \%$, with an $8.3 \%$ rate of over-staging and $29 \%$ rate of under-staging (35). MRI is limited by poor standardization of techniques and interpretation (36). With regard to PET/ CT, Kibel et al. evaluated [18F] fluorodeoxyglucose (FDG)$\mathrm{PET} / \mathrm{CT}$ in $42 \mathrm{cT} 2-3$ patients with negative conventional $\mathrm{CT}$ and bone scan, finding that FDG-PET/CT demonstrated a sensitivity of $70 \%$, specificity of $94 \%$, and positive predictive value of $78 \%$ (37). Another study recently found that altering the maximum standardized uptake value (SUVmax) of FDG-PET/CT resulted in varying sensitivity/ specificity profiles, with sensitivity/specificity of $79 \% / 66 \%$ for SUVmax $>2$, and sensitivity/specificity of $61 \% / 84 \%$ for SUVmax $>4$ (38). The diagnostic efficacy of [11C] choline $\mathrm{PET} / \mathrm{CT}$ was assessed in 44 patients who underwent $\mathrm{RC}$ in a German center, showing that accuracy with PET/CT was only $64 \%$ when compared to $61 \%$ for conventional CT (39).

Importantly, PLND should also be performed in conjunction with consolidative RC for patients receiving neoadjuvant chemotherapy. In fact, Herr et al. demonstrated that $92 \%$ of patients with regionally metastatic or unresectable bladder cancer who experienced major response to induction chemotherapy but did not undergo consolidative surgery died within 3 years. Moreover, Zargar-Shoshtari and colleagues assessed 304 patients with clinically node-positive disease who underwent neoadjuvant chemotherapy and showed that ypN0 was achieved in $48 \%$ (40). Of note, we recommend nodal biopsy in patients with clinical lymphadenopathy, in order to confirm histology and potentially guide treatment. Indeed, Moschini et al. evaluated 196 patients with clinical lymphadenopathy who underwent RC without neoadjuvant chemotherapy and found an $18 \%$ sensitivity for pathologic nodal involvement (41).

With regard to PLND during minimally-invasive approaches to RC, the same oncologic principles should be adhered to as in open surgery. Utilization of robotic $\mathrm{RC}$ has increased considerably, with a recent National Cancer Data Base analysis showing increase in frequency from $26 \%$ in 2010 to $39 \%$ in 2013 (42). Desai and colleagues have demonstrated feasibility of robotic PLND dissection to the aortic bifurcation and even the inferior mesenteric artery (IMA) (43). Abaza et al. performed a retrospective comparison of 120 open and 35 robotic cystectomies with extended PLND including pre-sacral lymphadenectomy and observed similar lymph node yield ( 37 versus $38 ; \mathrm{P}=0.68$ ) and pathologic node-positive rate $(30 \%$ versus $34 \% ; \mathrm{P}=0.73)$ between groups (44). Likewise, two randomized controlled trials of minimallyinvasive versus open RC, CORAL and RAZOR, found no difference in lymph node yield between approaches $(45,46)$. This observation was confirmed in a systematic review, which reported an average lymph node yield of 19 nodes and node-positive rate of $22 \%$ for robotic RC, with no significant difference in lymph node yield compared to the open approach (47).

Importantly, as with all steps in management of bladder cancer, competing comorbidities should be incorporated into the decision for and extent of PLND. For example, previous vascular stenting or renal transplantation may limit the ability to safely complete an extended dissection. A SEER-Medicare analysis by Novara et al. assessed 3,314 patients with RC alone versus RC and PLND, stratified survival by age and comorbidity subsets, and found that there was no difference in survival for patients older than 75 and with a Charlson-Comorbidity Index of 1 or greater based on performance of PLND (48). An example of this principle was demonstrated by Froehner et al., who showed that in 735 patients who underwent RC, those with fewer lymph nodes removed had decreased OS but similar cancerspecific survival, suggesting that there were increased competing risks in patients with a limited number of lymph nodes removed (49).

\section{Anatomy of lymphatic drainage and mapping studies}

Colston and Leadbetter first identified isolated pelvic lymph node metastases in cadaveric studies which suggested a lymphatic spread of bladder cancer (50). Lymphatic 
drainage was subsequently evaluated in numerous surgical and lymphoscintigraphy mapping studies as reviewed herein. Abol-Enein and colleagues performed a singlecenter surgical mapping study of 200 patients with routine dissection up to the origin of the IMA and demonstrated that lymph nodes were positive in $24 \%$ of cases, and of those positive cases, were bilateral in 39\% (51). Furthermore, the investigators noted that removing only nodes in the pelvis, defined as internal iliac, external iliac, and obturator regions, would capture just $66 \%$ of the positive nodes, while additional dissection to the level of the aortic bifurcation to include common iliac and presacral nodes would clear $79 \%$ of positive nodes (51). Importantly, there were no cases of "skip" metastases, with positive nodes proximal to the common iliac arteries without concomitant involvement of obturator/internal iliac nodes. Leissner et al. qualified these findings in a multi-center study assessing 290 cystectomies with dissection to the level of the IMA and pathologic examination of 12 clearly-defined anatomic locations (52). This study showed that $6.6 \%$ of patients had positive lymph nodes identified proximal to the aortic bifurcation, and of these, no patients had lymph node metastases exclusively above the aortic bifurcation (52). However, in another $6.9 \%$ of patients, positive lymph nodes were found exclusively between the common iliac bifurcation and the aortic bifurcation (52). Notably, the location of a single positive lymph node was most commonly in the obturator packet (52). Meanwhile, Vazina et al., in 43 patients with node-positive disease, found that all patients with disease proximal to the common iliac artery (16\%) also had at least one positive node in the obturator or internal iliac artery locations (53). Furthermore, as study by Tarin et al. found that $6 \%$ of node-positive patients had "skip" lesions above the common iliac bifurcation, corroborating Leissner et al.'s results but not Abol-Enein et al.'s or Vizina et al.'s (23). These mapping studies are limited by inconsistent definitions of anatomic boundaries as well as unknown rates of incomplete resection.

Lymphoscintigraphy has been utilized to help identify nodal landing sites. A study by Roth et al. in 2010 utilized single-photon emission CT (SPECT) with six bladder injections of technetium Tc $99 \mathrm{~m}$ (54). On subsequent extended PLND to the proximal level of uretero-iliac junction, 24 primary lymphatic landing sites per bladder and highly variable lymphatic patterns between patients were noted. The investigators concluded that dissection limited to the ventral external iliac artery and obturator packet would miss $48 \%$ of radiotracer positive nodes, while only $8 \%$ of radiotracer-positive lymph nodes were found proximal to the uretero-iliac junction (54). The authors also identified bilateral lymphatic spread in $15 \%$ of patients following unilateral bladder injections of radiotracer in a later study (55). Collectively, from these mapping studies, several tenets emerge: (I) lymph node involvement is frequently bilateral; (II) there is a small but consistent proportion of lymph nodes involved above of the true pelvis, and (III) while isolated common iliac nodes may be found in approximately $6 \%$ of patients, "skip" metastases above the aortic bifurcation without involvement of pelvic lymph nodes appear to be quite rare.

\section{PLND template nomenclature}

As reported in previous systematic reviews, no standard definitions exist for the extent of PLND at the time of RC (9,56). A variety of classifications have been reported: Level I, II, and III; as well as limited, standard, extended, and super-extended. The level classification was coined by Leissner and colleagues in 2004 (52). In this system, Level I nodes include obturator, deep obturator, internal and external iliac lymphatics, with proximal extent to the common iliac bifurcation. Level II includes lymphatics lateral to the common iliac arteries, pre-sacral nodes, and extends proximally to the aortic bifurcation. Level III nodes include paracaval, interaortocaval, and para-aortic lymphatics to the root of the IMA. From reported series, the boundaries of standard PLND dissection have typically extended from the genitofemoral nerve laterally to the bladder wall medially, distally to the node of Cloquet or superficial circumflex vein, and proximally to the bifurcation of the common iliac artery (26,57). Descriptions of extended PLND templates have been quite heterogeneous, with a proximal boundary extending to the uretero-iliac junction, aortic bifurcation, or various length cutoffs of the common iliac artery, and with variable inclusion of presacral nodes $(9,26)$. Meanwhile, super-extended templates have fairly consistently included resection of lymphatics up to the root of the IMA $(58,59)$. Given such variability, an expert panel from the European Association of Urology (EAU) Working Group on Muscle-Invasive Bladder Cancer defined the boundaries of dissection for a systematic review by Bruins et al. in 2014 (9). These templates are defined as follows: limited node dissection is that confined to the obturator and/or peri-vesical fossa only; standard template extends proximally to the common iliac arteries; extended template extends proximally to the crossing of the common iliac 
vessels with the ureters or to the aortic bifurcation, with or without the presacral LNs; super-extended template continues to the IMA.

\section{Lymph node count versus PLND template}

Various metrics have been described and reported to assess the adequacy of PLND. The two most common are lymph node count, which may in turn be used to calculate parameters such as lymph node density, as well as PLND template. With regard to lymph node count, the total number of nodes removed during PLND has been used as a surrogate for the quality of lymphadenectomy. An increasing number of nodes removed has moreover been associated with improved survival outcomes. Various specific node yield cutoffs have been put forth to represent an adequate PLND. In an early study by Herr et al., dichotomization of node-negative patients with $>8$ versus $<8$ nodes and node-positive patients with $>11$ or $<11$ nodes was associated with survival differences (18). In a subsequent series, Herr identified $>14$ nodes to be an optimal cutoff, with 5 -year OS rates of $33 \%, 44 \%, 73 \%$, and $79 \%$ for patients with $0-5,6-10,11-14$, and $>14$ nodes removed, respectively (60). Furthermore, in a re-analysis of SWOG 8710 data controlling for age, pathologic stage, node status, margins, and neoadjuvant chemotherapy administration, removal of $<10$ nodes was associated with a significantly increased post-cystectomy mortality (HR 2.0; $95 \%$ CI, 1.4-2.8; $\mathrm{P}=0.0001)$ (61). Meanwhile, Leissner and colleagues reported that the ideal threshold node count was 16 (17). Likewise, May et al. assessed an exclusively node-negative cohort and demonstrated that patients with $>16$ lymph nodes dissected had a 5 -year cancer-specific survival of $83 \%$ versus $72 \%$ for those with $<16$ lymph nodes removed (62). Using SEER data in 1,923 RC patients, Konety et al. demonstrated that increasing node yield generally yielded progressive improvement in survival for all stage subsets, but particularly for stage III node-positive patients (19). Whether these collective data in fact reflect a benefit to more extended dissection eliminating micro-metastatic disease versus simply improved staging (i.e., the "Will Rogers phenomenon") cannot be definitively established (33).

In addition, it is important to acknowledge that the use of a lymph node count is subject to a number of variables other than the extent of dissection. One problem is that there is simply large variability in the number of nodes present in different patients, as shown in an autopsy study that demonstrated a node yield ranging from 8 to 56 for standard template PLND in 30 cadavers (63). Furthermore, the surgeons who perform more thorough PLND may be more experienced and offer a higher quality RC operation than those generally performing limited dissections. Additionally, the institutions that these surgeons operate in may have superior process of care, which has been shown to improve outcomes (64). The reported node yield is also in the control of the pathologist assessing the lymphadenectomy specimen $(65,66)$. The pathologist protocol for examining lymphadenectomy specimens was shown to dramatically influence reported node yield in a study by Fang et al. (65). After institution of a policy to re-review specimens with less than 16 nodes by a more experienced pathologist, the reported median node yields jumped from 15 to 20. Similarly, Bochner et al. showed that submission of nodes from extended PLND en bloc yielded significantly lower reported node yield compared to submission of nodes in discrete anatomic packets (23 versus 36 nodes, respectively).

An alternative approach to assessing the extent of PLND is by reporting the template of dissection performed. With this approach, a beneficial effect of PLND has also been noted in observational studies $(67,68)$. Abdollah et al. utilized the SEER database to compare 11,183 patients who underwent RC with PLND versus without PLND between 1988 to 2006 (67). Patients with PLND had improved 10 -year cancer-specific survival and OS compared to those without PLND among all pathologic tumor stages. Several series have compared extended or super-extended to limited PLND templates (69-71) (Table 1). Holmer et al. found that in 170 patients there was significant improvement in disease specific survival after age, sex, tumor stage, lymph node status, and adjuvant chemotherapy were controlled for in multivariate analysis (HR 0.47; 95\% CI, 0.25-0.88; $\mathrm{P}=0.018$ ) (70). Jensen and colleagues studied 429 patients who underwent extended or limited PLND and found significant improvements in recurrence free survival (RFS) for the extended PLND group for both pathologic node-negative and node-positive subsets (69). Dhar et al. compared 336 RC patients that underwent limited PLND at Cleveland Clinic and 322 patients that underwent extended PLND at University of Bern (71). Extended PLND conferred significant benefit in 5-year RFS across multiple subsets including pT2N0 (77\% versus 67\%), pT3N0 (57\% versus $23 \%)$, pT2Nany (71\% versus $63 \%)$, pT3Nany (49\% versus $19 \%)$, p TanyN1 (35\% versus $7 \%$ ) (71). Several observational studies have further confirmed improved oncologic outcomes in patients undergoing extended or 


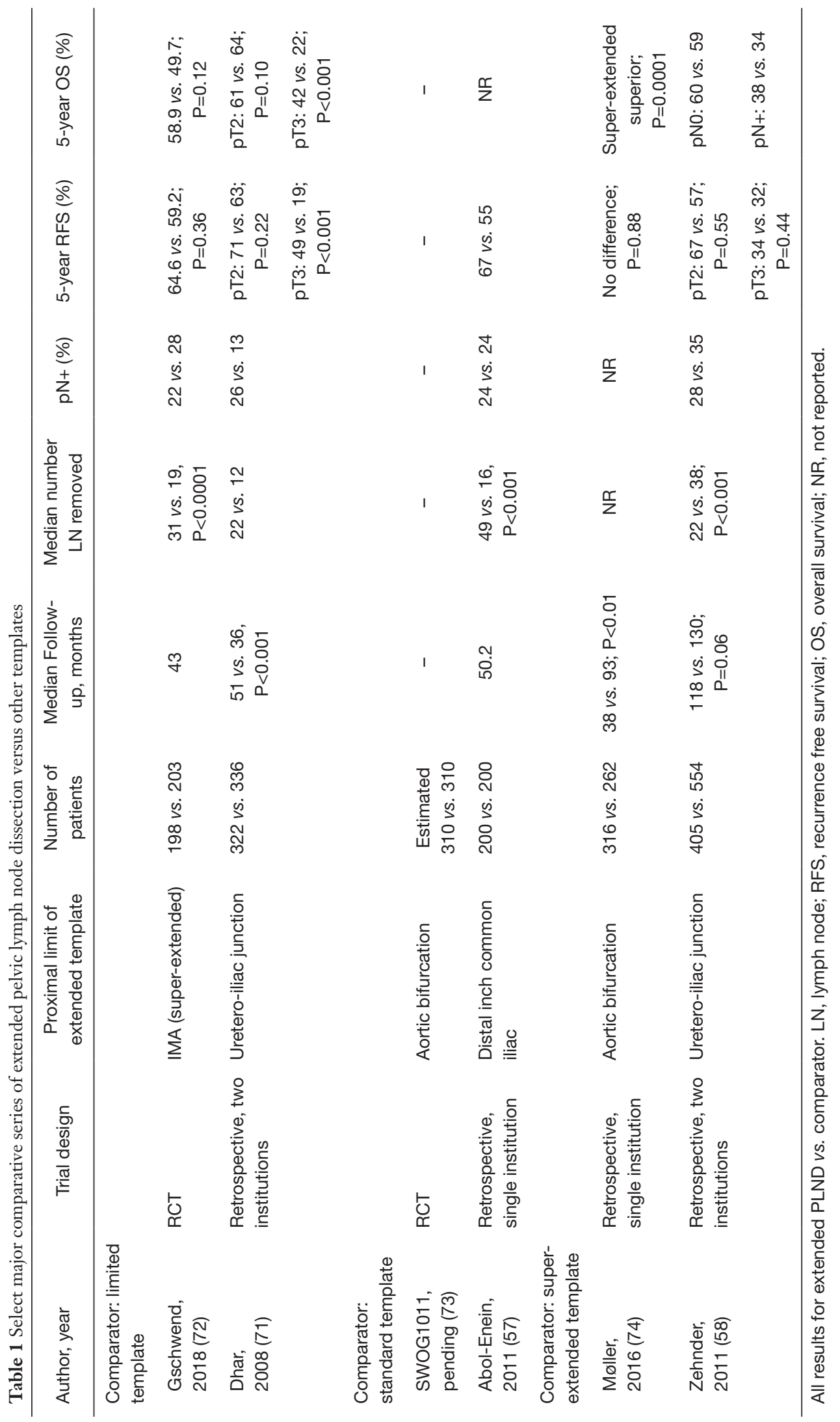


super-extended versus standard PLND (57,75-77). AbolEnein and colleagues assessed outcomes of 400 patients who underwent super-extended versus standard PLND at the discretion of two surgeons at a single institution (57). This report demonstrated a significant improvement in 5-year disease-specific survival in patients with extended versus standard PLND (48\% versus $28 \%$ ). While there have been mixed results in other analyses of these templates, these results are from smaller series or incomplete publications (9). Super-extended versus extended PLND templates have likewise been compared $(58,74)$. Zehnder et al. evaluated 554 University of Southern California patients who underwent super-extended PLND and 405 University of Bern patients who underwent extended PLND between 1985 to 2005 with no neoadjuvant chemotherapy, negative margins, and pT2/3 disease (58). Extended PLND included a proximal extent to the mid-upper third of the common iliac artery and super-extended PLND included all nodes up to the IMA. Super-extended dissection yielded a higher nodal yield (38 versus 22) and higher rate of lymph nodes metastases (35\% versus 28\%) (58). Nevertheless, there was almost identical 5-year distant disease-free survival for super-extended versus extended groups $(65 \%$ versus $66 \%$ ) regardless of stratification by tumor stage or node positivity (58). Møller et al. also compared superextended versus extended PLND in 578 patients and found similar recurrence-free survival between groups (74).

In summary, the aforementioned existing observational data seems to suggest that performing a PLND is associated with improved outcomes compared to not performing a PLND, while an increasing extent of PLND, assessed either by lymph node yield or extent of template, is likely to be of benefit, but may reach a threshold above which further dissection does not incrementally enhance disease control. However, there are major limitations to these data that preclude clear recommendations regarding extent of PLND template, which has been noted by several systematic reviews on this topic $(9,56)$. The majority of studies that compare extended or super-extended PLND to smaller templates have significant selection bias and confounding as templates were often not designated prospectively, left to surgeon preference, and amended intra-operatively. Furthermore, these studies were non-randomized and were evaluated in a retrospective fashion.

\section{Clinical trials assessing PLND template}

Two recent randomized controlled trials have evaluated the association of extent of PLND with oncologic outcomes. The LEA trial, conducted by the Association of Urogenital Oncology of the German Cancer Society in 16 German centers from 2006 to 2010, compared extended versus limited PLND (72). Patients with T1G3-T4a bladder cancer who did not receive neoadjuvant chemotherapy were included. A total of 401 patients were randomized 1:1 to either extended or limited PLND, with limited PLND including obturator, internal, and external iliac nodes and extended PLND additionally including deep obturator, common iliac, presacral, paracaval, interaortocaval, and para-aortal nodes up to the IMA. Notably, the template referred to by the trial as an extended template is considered super-extended by other definitions. The study demonstrated a significantly higher nodal yield (31 versus $19, \mathrm{P}<0.001)$ and numerically higher rate of lymph node metastases ( $28 \%$ versus $22 \% ; \mathrm{P}=0.12)$ in the extended versus limited PLND group. However, compared to a limited PLND, extended PLND was not associated with statistically significant improved 5-year recurrence-free survival ( $65 \%$ versus $59 \% ; \mathrm{P}=0.36$ ), cancer-specific survival (76\% versus $65 \% ; \mathrm{P}=0.10)$, or OS $(59 \%$ versus $50 \%$; $\mathrm{P}=0.12)$. There was no difference in 90 -day complication rates between groups, although extended PLND resulted in a higher rate of lymphoceles requiring intervention $(8.6 \%$ versus $3.4 \%$; $\mathrm{P}=0.04)$. Importantly, the study was powered to detect a $15 \%$ difference in recurrence-free survival and therefore may have been underpowered to detect a true but smaller difference between groups. Furthermore, the inclusion of clinical T1G3 tumors and the finding that only one patient had pathologic N3 disease indicate a relatively low risk of the overall cohort. Thus, while the study was reported negative, it may have additionally been underpowered by its low risk population to detect a benefit from extended PLND.

S-1011 was a randomized trial run by SWOG that also compared standard versus extended PLND (73). A total of 620 patients were accrued between 2011 and 2017. The standard PLND template included peri-vesical, obturator, internal and external iliac lymph nodes up to the common iliac bifurcation. The extended template additionally included common iliac nodes to the bifurcation of the aorta as well as pre-sacral nodes. The study was powered to demonstrate $10 \%$ improvement in 3 -year disease-free survival, and these data are estimated to mature by 2022 . Important differences from the completed LEA trial include allowance for neoadjuvant chemotherapy, which is a stratification factor. Additionally, only patients with at least 
clinical stage T2 disease were included. Finally, there is a less extensive proximal limit of dissection in the extended PLND group in this trial.

\section{Complications from PLND}

Relatively minimal additional morbidity secondary to the performance of PLND has been reported. While hemorrhage and nerve injury are unique side effects that can occur as lymphatics are skeletonized off the iliac vessels and obturator nerve, these are reported quite infrequently in the literature (9). One potential sequalae of PLND is pelvic lymphocele formation. Lymphoceles represent a potential source of infection, and may compress iliac venous drainage, increasing the risk of lower extremity thrombosis. Lymphocele risk is likely related to the extent if dissection, as lymphoceles requiring drainage occurred at a higher rate in the extended PLND arm of the LEA trial (72). Other overall complications appear similar across increasing extent of dissection (78). Nevertheless, several comparative series have noted an approximately extra $60 \mathrm{~min}$ of operative time for extended versus limited PLND for both open and robotic approaches (77-79). This factor may be considered when deciding the extent of PLND in a patient with significant medical comorbidities for whom additional time under anesthesia would be of potential concern.

\section{Future developments}

The variability of initial lymphatic spread demonstrated in classic anatomic mapping studies require the detection of tracers throughout the pelvis, rather than limited to a single group of nodes as in breast cancer (52). The use of radiotracer and fluorescent markers to guide sentinel node dissections for bladder cancer have not yet shown reproducible and reliable outcomes. The use of radiotracers has had mixed results. A small series by Rosenblatt et al. reported a detection rate of $92 \%$ using peritumoral injection with Technetium tracers (80). However, another series by Aljabery et al. used peritumoral Nanacoll injections and demonstrated a sensitivity of only $67 \%$ (81). Meanwhile, a study by Polom $e t$ al. included both technetium radiocolloid (RadCol) and indocyanine green (ICG) cystoscopic peritumoral injections in 47 patients prior to RC (82). Pre-operative SPECT/CT and intra-operative gamma ray detection probes were used to assess RadCol while the robotic near-infrared fluorescent (NIRF) camera system was used to assess ICG. The ICG method was found to reveal more nodes than RadCol; nevertheless, only $17 \%$ of patients with ICG fluorescence were truly metastatic. Patel et al. also assessed the feasibility of ICG and observed that in patients with ICG nodal fluorescence there was only $47 \%$ specificity (83).

Of note, as systemic therapy options for bladder cancer continue to expand, the role and timing of RC with PLND will require continued study. For example, the novel checkpoint inhibitor agents theoretically require an appropriate local tumor microenvironment and/or lymphatic milieu to allow tumor antigen-specific priming of cytotoxic $\mathrm{T}$ cells, and as such may benefit from preservation of both primary tumor and pelvic lymph nodes (84). To this end, there are a number of clinical trials investigating the utility of neoadjuvant checkpoint inhibition prior to RC (85). Early reports of these trials show promising rates of compete pathologic response, although longer-term follow-up for survival outcomes will be needed (86).

\section{Conclusions}

PLND during RC affords accurate pathologic stage, provides information on prognosis, facilitates timely consideration of adjuvant therapy, and potentially may confer oncologic benefit. Admittedly, the current evidence is mixed regarding whether an increasing extent of PLND results in improved survival. While the majority of studies to date on this topic have a low-quality of evidence, forthcoming prospective clinical trial data will offer opportunities to inform future lymphadenectomy guidelines for bladder cancer.

\section{Acknowledgments}

Funding: None.

\section{Footnote}

Provenance and Peer Review: This article was commissioned by the Guest Editor (Shomik Sengupta) for the series "Surgery for Urologic Cancers" published in Translational Andrology and Urology. The article has undergone external peer review.

Conflicts of Interest: All authors have completed the ICMJE uniform disclosure form (available at http://dx.doi. 
org/10.21037/tau.2019.06.01). The series "Surgery for Urologic Cancers" was commissioned by the editorial office without any funding or sponsorship. Dr. Boorjian reports other from Ferring, other from Sanofi, other from ArTara, outside the submitted work. The authors have no other conflicts of interest to declare.

Ethical Statement: The authors are accountable for all aspects of the work in ensuring that questions related to the accuracy or integrity of any part of the work are appropriately investigated and resolved.

Open Access Statement: This is an Open Access article distributed in accordance with the Creative Commons Attribution-NonCommercial-NoDerivs 4.0 International License (CC BY-NC-ND 4.0), which permits the noncommercial replication and distribution of the article with the strict proviso that no changes or edits are made and the original work is properly cited (including links to both the formal publication through the relevant DOI and the license). See: https://creativecommons.org/licenses/by-nc-nd/4.0/.

\section{References}

1. Bray F, Ferlay J, Soerjomataram I, et al. Global cancer statistics 2018: GLOBOCAN estimates of incidence and mortality worldwide for 36 cancers in 185 countries. CA Cancer J Clin 2018;68:394-424.

2. Kaufman DS, Shipley WU, Feldman AS. Bladder cancer. Lancet 2009;374:239-49.

3. Clark PE, Agarwal N, Biagioli MC, et al. Bladder cancer. J Natl Compr Canc Netw 2013;11:446-75.

4. Chang SS, Bochner BH, Chou R, et al. Treatment of NonMetastatic Muscle-Invasive Bladder Cancer: AUA/ASCO/ ASTRO/SUO Guideline. J Urol 2017;198:552-9.

5. Chang SS, Boorjian SA, Chou R, et al. Diagnosis and Treatment of Non-Muscle Invasive Bladder Cancer: AUA/ SUO Guideline. J Urol 2016;196:1021-9.

6. Babjuk M, Burger M, Zigeuner R, et al. EAU Guidelines on Non-Muscle-invasive Urothelial Carcinoma of the Bladder: Update 2013. Eur Urol 2013;64:639-53.

7. Canter D, Long C, Kutikov A, et al. Clinicopathological outcomes after radical cystectomy for clinical $\mathrm{T} 2$ urothelial carcinoma: further evidence to support the use of neoadjuvant chemotherapy. BJU Int 2011;107:58-62.

8. Bruins HM, Skinner EC, Dorin RP, et al. Incidence and location of lymph node metastases in patients undergoing radical cystectomy for clinical non-muscle invasive bladder cancer: results from a prospective lymph node mapping study. Urol Oncol 2014;32:24.e13-9.

9. Bruins HM, Veskimae E, Hernandez V, et al. The impact of the extent of lymphadenectomy on oncologic outcomes in patients undergoing radical cystectomy for bladder cancer: a systematic review. Eur Urol 2014;66:1065-77.

10. Perera $M, M c G r a t h S$, Sengupta $S$, et al. Pelvic lymph node dissection during radical cystectomy for muscleinvasive bladder cancer. Nat Rev Urol 2018;15:686-92.

11. Lobo N, Mount C, Omar K, et al. Landmarks in the treatment of muscle-invasive bladder cancer. Nat Rev Urol 2017; 14:565-74.

12. Leadbetter WF, Cooper JF. Regional gland dissection for carcinoma of the bladder; a technique for one-stage cystectomy, gland dissection, and bilateral ureteroenterostomy. J Urol 1950;63:242-60.

13. Whitmore WF, Marshall VF. Radical Total Cystectomy for Cancer of the Bladder: 230 Consecutive Cases Five Years Later. J Urol 1962;87:853-68.

14. Dretler SP, Ragsdale BD, Leadbetter WF. The value of pelvic lymphadenectomy in the surgical treatment of bladder cancer. J Urol 1973;109:414-6.

15. Skinner DG. Management of Invasive Bladder Cancer: A Meticulous Pelvic Node Dissection Can Make a Difference. J Urol 1982;128:34-6.

16. Cole AP, Dalela D, Hanske J, et al. Temporal trends in receipt of adequate lymphadenectomy in bladder cancer 1988 to 2010. Urol Oncol 2015;33:504.e9-17.

17. Leissner J, Hohenfellner R, Thüroff JW, et al. Lymphadenectomy in patients with transitional cell carcinoma of the urinary bladder; significance for staging and prognosis. BJU Int 2000;85:817-23.

18. Herr HW, Bochner BH, Dalbagni G, et al. Impact of the Number of Lymph Nodes Retreived on Outcome in Paitents with Muscle-Invasive Bladder Cancer. J Urol 2002;167:1295-8.

19. Konety BR, Joslyn SA, O'Donnell MA. Extent of Pelvic Lymphadenectomy and Its Impact On Outcome in Patients Diagnosed With Bladder Cancer: Analysis of Data From the Surveillance, Epidemiology and End Results Program Data Base. J Urol 2003;169:946-50.

20. Wright JL, Lin DW, Porter MP. The association between extent of lymphadenectomy and survival among patients with lymph node metastases undergoing radical cystectomy. Cancer 2008;112:2401-8.

21. Brierley JD, Gospodarowicz MK, Wittekind C, editors. TNM classification of malignant tumours. Eighth edition. Chichester: John Wiley \& Sons, Ltd., 2017. 
22. Paner GP, Stadler WM, Hansel DE, et al. Updates in the Eighth Edition of the Tumor-Node-Metastasis Staging Classification for Urologic Cancers. Eur Urol 2018;73:560-9.

23. Tarin TV, Power NE, Ehdaie B, et al. Lymph NodePositive Bladder Cancer Treated With Radical Cystectomy and Lymphadenectomy: Effect of the Level of Node Positivity. Eur Urol 2012;61:1025-30.

24. Bruins HM, Dorin RP, Rubino B, et al. Critical evaluation of the American Joint Committee on Cancer TNM nodal staging system in patients with lymph node-positive disease after radical cystectomy. Eur Urol 2012;62:671-6.

25. International Bladder Cancer Nomogram Consortium, Bochner BH, Kattan MW, et al. Postoperative nomogram predicting risk of recurrence after radical cystectomy for bladder cancer. J Clin Oncol 2006;24:3967-72.

26. Herr H, Lee C, Chang S, et al. Standardization of Radical Cystectomy and Pelvic Lymph Node Dissection for Bladder Cancer: A Collaborative Group Report. J Urol 2004;171:1823-8.

27. Herr HW. Superiority of Ratio Based Lymph Node Staging for Bladder Cancer. J Urol 2003;169:943-5.

28. Ku JH, Kang M, Kim HS, et al. Lymph node density as a prognostic variable in node-positive bladder cancer: a meta-analysis. BMC Cancer 2015;15:447.

29. Fajkovic H, Cha EK, Jeldres C, et al. Extranodal extension is a powerful prognostic factor in bladder cancer patients with lymph node metastasis. Eur Urol 2013;64:837-45.

30. Fleischmann A, Thalmann GN, Markwalder R, et al. Extracapsular extension of pelvic lymph node metastases from urothelial carcinoma of the bladder is an independent prognostic factor. J Clin Oncol 2005;23:2358-65.

31. Ahn TS, Kim HS, Jeong CW, et al. Extracapsular Extension of Pelvic Lymph Node Metastasis is an Independent Prognostic Factor in Bladder Cancer: A Systematic Review and Meta-analysis. Ann Surg Oncol 2015;22:3745-50.

32. Koppie TM, Vickers AJ, Vora K, et al. Standardization of pelvic lymphadenectomy performed at radical cystectomy: Can we establish a minimum number of lymph nodes that should be removed? Cancer 2006;107:2368-74.

33. Gofrit ON, Zorn KC, Steinberg GD, et al. The Will Rogers Phenomenon in Urological Oncology. J Urol 2008;179:28-33.

34. Moschini M, Karnes RJ, Gandaglia G, et al. Preoperative Favorable Characteristics in Bladder Cancer Patients Cannot Substitute the Necessity of Extended Lymphadenectomy During Radical Cystectomy: A
Sensitivity Curve Analysis. Urology 2016;88:97-103.

35. Tritschler S, Mosler C, Straub J, et al. Staging of muscleinvasive bladder cancer: can computerized tomography help us to decide on local treatment? World J Urol 2012;30:827-31.

36. Giannarini G, Petralia G, Thoeny HC. Potential and Limitations of Diffusion-Weighted Magnetic Resonance Imaging in Kidney, Prostate, and Bladder Cancer Including Pelvic Lymph Node Staging: A Critical Analysis of the Literature. Eur Urol 2012;61:326-40.

37. Kibel AS, Dehdashti F, Katz MD, et al. Prospective study of $[18 \mathrm{~F}]$ fluorodeoxyglucose positron emission tomography/computed tomography for staging of muscle-invasive bladder carcinoma. J Clin Oncol 2009;27:4314-20.

38. Vind-Kezunovic S, Bouchelouche K, Ipsen P, et al. Detection of Lymph Node Metastasis in Patients with Bladder Cancer using Maximum Standardised Uptake Value and 18F-fluorodeoxyglucose Positron Emission Tomography/Computed Tomography: Results from a High-volume Centre Including Long-term Follow-up. Eur Urol Focus 2019;5:90-6.

39. Maurer T, Souvatzoglou M, Kübler H, et al. Diagnostic Efficacy of [11C]Choline Positron Emission Tomography/ Computed Tomography Compared With Conventional Computed Tomography in Lymph Node Staging of Patients With Bladder Cancer Prior to Radical Cystectomy. Eur Urol 2012;61:1031-8.

40. Zargar-Shoshtari K, Zargar H, Lotan Y, et al. A MultiInstitutional Analysis of Outcomes of Patients with Clinically Node Positive Urothelial Bladder Cancer Treated with Induction Chemotherapy and Radical Cystectomy. J Urol 2016;195:53-9.

41. Moschini M, Morlacco A, Briganti A, et al. Clinical Lymphadenopathy in Urothelial Cancer: A Transatlantic Collaboration on Performance of Cross-sectional Imaging and Oncologic Outcomes in Patients Treated with Radical Cystectomy Without Neoadjuvant Chemotherapy. Eur Urol Focus 2018;4:245-51.

42. Bachman AG, Parker AA, Shaw MD, et al. Minimally Invasive Versus Open Approach for Cystectomy: Trends in the Utilization and Demographic or Clinical Predictors Using the National Cancer Database. Urology 2017;103:99-105.

43. Desai MM, Berger AK, Brandina RR, et al. Robotic and Laparoscopic High Extended Pelvic Lymph Node Dissection During Radical Cystectomy: Technique and Outcomes. Eur Urol 2012;61:350-5. 
44. Abaza R, Dangle PP, Gong MC, et al. Quality of Lymphadenectomy is Equivalent With Robotic and Open Cystectomy Using an Extended Template. J Urol 2012;187:1200-4.

45. Bochner BH, Dalbagni G, Sjoberg DD, et al. Comparing Open Radical Cystectomy and Robot-assisted Laparoscopic Radical Cystectomy: A Randomized Clinical Trial. Eur Urol 2015;67:1042-50.

46. Parekh DJ, Reis IM, Castle EP, et al. Robot-assisted radical cystectomy versus open radical cystectomy in patients with bladder cancer (RAZOR): an open-label, randomised, phase 3, non-inferiority trial. Lancet 2018;391:2525-36.

47. Yuh B, Artibani W, Heidenreich A, et al. The role of robot-assisted radical prostatectomy and pelvic lymph node dissection in the management of high-risk prostate cancer: a systematic review. Eur Urol 2014;65:918-27.

48. Novara G, Catto JWF, Wilson T, et al. Systematic Review and Cumulative Analysis of Perioperative Outcomes and Complications After Robot-assisted Radical Cystectomy. Eur Urol 2015;67:376-401.

49. Froehner M, Novotny V, Heberling U, et al. Relationship of the Number of Removed Lymph Nodes to Bladder Cancer and Competing Mortality After Radical Cystectomy. Eur Urol 2014;66:987-90.

50. Colston JAC, Leadbetter WF. Infiltrating Carcinoma of the Bladder. J Urol 1936;36:669-83.

51. Abol-Enein H, El-Baz M, Abd El-Hameed MA, et al. Lymph Node Involvement in Patients with Bladder Cancer Treated with Radical Cystectomy: A PathoAnatomical Study-A Single Center Experience. J Urol 2004;172:1818-21.

52. Leissner J, Ghoneim MA, Abol-Enein H, et al. Extended Radical Lymphadenectomy in Patients With Urothelial Bladder Cancer:: Results of a Prospective Multicenter Study. J Urol 2004;171:139-44.

53. Vazina A, Dugi D, Shariat SF, et al. Stage Specific Lymph Node Metastasis Mapping in Radical Cystectomy Specimens. J Urol 2004;171:1830-4.

54. Roth B, Wissmeyer MP, Zehnder P, et al. A New Multimodality Technique Accurately Maps the Primary Lymphatic Landing Sites of the Bladder. Eur Urol 2010;57:205-11.

55. Roth B, Zehnder P, Birkhäuser FD, et al. Is Bilateral Extended Pelvic Lymphadenectomy Necessary for Strictly Unilateral Invasive Bladder Cancer? J Urol 2012;187:1577-82.

56. Bi L, Huang H, Fan X, et al. Extended vs non-extended pelvic lymph node dissection and their influence on recurrence-free survival in patients undergoing radical cystectomy for bladder cancer: a systematic review and meta-analysis of comparative studies: Meta-analysis of studies on extended vs non-extended PLND. BJU Int 2014;113:E39-48.

57. Abol-Enein H, Tilki D, Mosbah A, et al. Does the Extent of Lymphadenectomy in Radical Cystectomy for Bladder Cancer Influence Disease-Free Survival? A Prospective Single-Center Study. Eur Urol 2011;60:572-7.

58. Zehnder P, Studer UE, Skinner EC, et al. Super Extended Versus Extended Pelvic Lymph Node Dissection in Patients Undergoing Radical Cystectomy for Bladder Cancer: A Comparative Study. J Urol 2011;186:1261-8.

59. Dorin RP, Daneshmand S, Eisenberg MS, et al. Lymph Node Dissection Technique Is More Important Than Lymph Node Count in Identifying Nodal Metastases in Radical Cystectomy Patients: A Comparative Mapping Study. Eur Urol 2011;60:946-52.

60. Herr HW. Extent of surgery and pathology evaluation has an impact on bladder cancer outcomes after radical cystectomy. Urology 2003;61:105-8.

61. Herr HW, Faulkner JR, Grossman HB, et al. Surgical Factors Influence Bladder Cancer Outcomes: A Cooperative Group Report. J Clin Oncol 2004;22:2781-9.

62. May M, Herrmann E, Bolenz C, et al. Association Between the Number of Dissected Lymph Nodes During Pelvic Lymphadenectomy and Cancer-Specific Survival in Patients with Lymph Node-Negative Urothelial Carcinoma of the Bladder Undergoing Radical Cystectomy. Ann Surg Oncol 2011;18:2018-25.

63. Weingärtner K, Ramaswamy A, Bittinger A, et al. Anatomical Basis for Pelvic Lymphadenectomy in Prostate Cancer: Results of an Autopsy Study and Implications for the Clinic. J Urol 1996;156:1969-71.

64. Hollenbeck BK, Wei Y, Birkmeyer JD. Volume, Process of Care, and Operative Mortality for Cystectomy for Bladder Cancer. Urology 2007;69:871-5.

65. Fang AC, Ahmad AE, Whitson JM, et al. Effect of a minimum lymph node policy in radical cystectomy and pelvic lymphadenectomy on lymph node yields, lymph node positivity rates, lymph node density, and survivorship in patients with bladder cancer. Cancer 2010;116:1901-8.

66. Bochner BH, Herr HW, Reuter VE. Impact of separate versus en bloc pelvic lymph node dissection on the number of lymph nodes retrieved in cystectomy specimens. J Urol 2001;166:2295-6.

67. Abdollah F, Sun M, Schmitges J, et al. Stage-specific impact of pelvic lymph node dissection on survival in 
patients with non-metastatic bladder cancer treated with radical cystectomy. BJU Int 2012;109:1147-54.

68. Brunocilla E, Pernetti R, Schiavina R, et al. The number of nodes removed as well as the template of the dissection is independently correlated to cancer-specific survival after radical cystectomy for muscle-invasive bladder cancer. Int Urol Nephrol 2013;45:711-9.

69. Jensen JB, Ulhøi BP, Jensen KM-E. Extended versus limited lymph node dissection in radical cystectomy: Impact on recurrence pattern and survival: Extended vs limited LND in RC. Int J Urol 2012;19:39-47.

70. Holmer M, Bendahl PO, Davidsson T, et al. Extended lymph node dissection in patients with urothelial cell carcinoma of the bladder: can it make a difference? World J Urol 2009;27:521-6.

71. Dhar NB, Klein EA, Reuther AM, et al. Outcome after radical cystectomy with limited or extended pelvic lymph node dissection. J Urol 2008;179:873-8; discussion 878.

72. Gschwend JE, Heck MM, Lehmann J, et al. Extended Versus Limited Lymph Node Dissection in Bladder Cancer Patients Undergoing Radical Cystectomy: Survival Results from a Prospective, Randomized Trial. Eur Urol 2019;75:604-11.

73. US National Library of Medicine. ClinicalTrials.gov. S1011 Standard or Extended Pelvic Lymphadenectomy in Treating Patients Undergoing Surgery for Invasive Bladder Cancer. 2018. Available online: https://clinicaltrials.gov/ ct2/show/NCT01224665

74. Møller MK, Høyer S, Jensen JB. Extended versus superextended lymph-node dissection in radical cystectomy: subgroup analysis of possible recurrence-free survival benefit. Scand J Urol 2016;50:175-80.

75. Simone G, Papalia R, Ferriero M, et al. Stage-specific impact of extended versus standard pelvic lymph node dissection in radical cystectomy: Node dissection in radical cystectomy. Int J Urol 2013;20:390-7.

76. Poulsen AL, Horn T, Steven K. Radical cystectomy: extending the limits of pelvic lymph node dissection improves survival for patients with bladder cancer confined to the bladder wall. J Urol 1998;160:2015-19.

77. Finelli A, Gill IS, Desai MM, et al. Laparoscopic extended pelvic lymphadenectomy for bladder cancer: technique and initial outcomes. J Urol 2004;172:1809-12.

78. Brössner C, Pycha A, Toth A, et al. Does extended lymphadenectomy increase the morbidity of radical cystectomy? BJU Int 2004;93:64-6.

79. Shao P, Meng X, Li J, et al. Laparoscopic extended pelvic lymph node dissection during radical cystectomy: technique and clinical outcomes. BJU Int 2011;108:124-8.

80. Rosenblatt R, Johansson M, Alamdari F, et al. Sentinel node detection in muscle-invasive urothelial bladder cancer is feasible after neoadjuvant chemotherapy in all pT stages, a prospective multicenter report. World J Urol 2017;35:921-7.

81. Aljabery F, Shabo I, Olsson H, et al. Radio-guided sentinel lymph node detection and lymph node mapping in invasive urinary bladder cancer: a prospective clinical study. BJU Int 2017;120:329-36.

82. Polom W, Markuszewski M, Cytawa W, et al. Fluorescent Versus Radioguided Lymph Node Mapping in Bladder Cancer. Clin Genitourin Cancer 2017;15:e405-9.

83. Patel MN, Hemal AK. Molecular Targeted FluorescenceGuided Intraoperative Imaging of Bladder Cancer Nodal Drainage Using Indocyanine Green During Radical and Partial Cystectomy. Curr Urol Rep 2016;17:74.

84. Chen DS, Mellman I. Oncology meets immunology: the cancer-immunity cycle. Immunity 2013;39:1-10.

85. Bellmunt J, Powles T, Vogelzang NJ. A review on the evolution of PD-1/PD-L1 immunotherapy for bladder cancer: The future is now. Cancer Treat Rev 2017;54:5867.

86. Necchi A, Anichini A, Raggi D, et al. Pembrolizumab as Neoadjuvant Therapy Before Radical Cystectomy in Patients With Muscle-Invasive Urothelial Bladder Carcinoma (PURE-01): An Open-Label, Single-Arm, Phase II Study. J Clin Oncol 2018;36:3353-60.
Cite this article as: Packiam VT, Tsivian M, Boorjian SA. The evolving role of lymphadenectomy for bladder cancer: why, when, and how. Transl Androl Urol 2020;9(6):3082-3093. doi: 10.21037/tau.2019.06.01 